\title{
A novel AP4M1 mutation in autosomal recessive cerebral palsy syndrome and clinical expansion of AP-4 deficiency
}

\author{
Muhammad Jameel', Joakim Klar², Muhammad Tariq ${ }^{1}$, Abubakar Moawia', Naveed Altaf Malik', \\ Syeda Seema Waseem¹, Uzma Abdullah', Tahir Naeem Khan', Raili Raininko³, Shahid Mahmood Baig ${ }^{1}$ \\ and Niklas Dahl ${ }^{2 *}$
}

\begin{abstract}
Background: Cerebral palsy (CP) is a heterogeneous neurodevelopmental disorder associated with intellectual disability in one-third of cases. Recent findings support Mendelian inheritance in subgroups of patients with the disease. The purpose of this study was to identify a novel genetic cause of paraplegic CP with intellectual disability in a consanguineous Pakistani family.

Methods: We performed whole-exome sequencing (WES) in two brothers with CP and intellectual disability. Analysis of AP4M1 mRNA was performed using quantitative real-time PCR on total RNA from cultured fibroblasts. The brothers were investigated clinically and by MRI.

Results: We identified a novel homozygous AP4M1 mutation c.194_195delAT, p.Y65Ffs*50 in the affected brothers. Quantitative RT-PCR analysis showed markedly reduced AP4M1 mRNA levels suggesting partial non-sense mediated mRNA decay. Several clinical and MRI features were consistent with AP-4 complex deficiency. However, in contrast to previously reported cases with AP4M1 mutations our patients show an aggressive behavior and a relatively late onset of disease.
\end{abstract}

Conclusion: This study shows an AP4M1 mutation associated with aggressive behavior in addition to mild dysmorphic features, intellectual disability, spastic paraparesis and reduced head circumference. Our findings expand the clinical spectrum associated with AP-4 complex deficiency and the study illustrates the importance of MRI and WES in the diagnosis of patients with CP and intellectual disability.

Keywords: Cerebral palsy, AP-4 deficiency, AP4M1 gene, Mutation, Clinical variability

\section{Background}

Cerebral palsy (CP) is a common cause of physical disability in childhood with an incidence of approximately $0.2-3 \%$ $[1,2]$. The causes of $\mathrm{CP}$ are heterogeneous and specific underlying causes remain unknown in the majority of cases. It is noteworthy that neonatal hypoxia, previously assumed to be a predominant cause of $\mathrm{CP}$, accounts for no more than $10-20 \%$ of cases [3,4]. In line with this, independent studies have provided evidences for genetic causes behind CP related disorders. This was suggested

\footnotetext{
* Correspondence: niklas.dahl@igp.uu.se

${ }^{2}$ Department of Immunology, Genetics and Pathology, Science for Life Laboratory, Uppsala University, Uppsala 751 08, Sweden

Full list of author information is available at the end of the article
}

already by an earlier twin-study showing an increased concordance rate for $\mathrm{CP}$ in monozygotic vs. dizygotic twins [5]. Furthermore, the association of CP with congenital malformations supported the hypothesis that genes may be involved in the disease [6,7]. More recent studies have confirmed that $\mathrm{CP}$ may be inherited as a Mendelian trait caused by single gene mutations in subgroups of patients. The four components of the adaptor protein 4 (AP-4) complex (reviewed by Moreno-De-Luca et al. [8]) have been linked to monogenic CP. The AP-4 hetero-tetramer is composed of the AP4B1, AP4M1, AP4E1 and AP4S1 subunits that are of critical importance for vesicular transport [9]. Thus, the AP-4 complex is required for appropriate intracellular transport as well as for 
secretion and endocytosis. Furthermore, the AP-4 complex sorts the AMPA glutamate receptors that are required for excitatory synaptic neurotransmission of importance during brain development [10]. To date, a limited number of families have been described segregating autosomal recessive CP that is caused by a mutation in either of the $A P 4 B 1, A P 4 E 1, A P 4 M 1$ and AP4S1 genes [8,11-15]. The different mutations presumably cause a disruption of the AP-4 complex integrity and the affected individuals share clinical characteristics including intellectual disability, reduced head circumference, short stature and spastic di- or paraplegia. Symptoms such as hypotonia and/or microcephaly may be present at birth or in the neonatal period but development may be within normal ranges up to several months. The disease progresses usually within the first 2 years of age (y.o.a.) with loss of acquired motor functions, peripheral neuropathy, spasticity and sometimes seizures $[14,15]$.

Here we report on a consanguineous family with two sons affected by a complex form of CP. The brothers had an onset at approximately 12 months of age (m.o.a) and they are homozygous for a novel and truncating AP4M1 gene mutation associated with markedly reduced AP4M1 mRNA levels. The clinical features in our patients bring further information on the variability and delineation of this subgroup of complex CP with AP-4 deficiency.

\section{Methods}

\section{Patients}

The consanguineous family is of Pakistani origin without family history of any neurological disease. The healthy parents are first cousins with four children (Figure 1A). Two sons have cerebral palsy, spastic paraplegia and intellectual disability whereas two sons are healthy. Informed and written consent was obtained from the parents being legal guardians of their sons. The study was carried out in accordance with the Declaration of Helsinki and the protocol approved by the local ethical committee, National Institute for Biotechnology and Genetic Engineering (NIBGE), > Faisalabad, Pakistan.

\section{Whole exome sequencing}

Whole exome sequencing (WES) was performed on $50 \mathrm{ng}$ of genomic DNA from the two affected brothers as described previously [16]. In brief, DNA was sheared by sonication with the Covaris S2 instrument (Covaris, Inc.). Fragment libraries were created from the sheared samples using the AB Library Builder System (Life Technologies) and target enrichment was performed according to the manufacturer's protocols (Agilent SureSelect Human All Exon v4 kit). Exome capture was conducted by hybridizing the DNA libraries with biotinylated RNA baits for $24 \mathrm{~h}$ followed by extraction using streptavidin coated magnetic beads. Captured DNA was then amplified followed by emulsion PCR using the EZ Bead System (Life Technologies) and sequenced on the SOLiD5500xl system, generating over 100 million reads of $75 \mathrm{bp}$ length for each of the samples.

Alignment of reads to the human reference sequence (hg19 assembly) and variant detection was performed using v2.1 of the LifeScope Software. SNPs and indel data was stored in an in-house exome database together with variant annotation information obtained

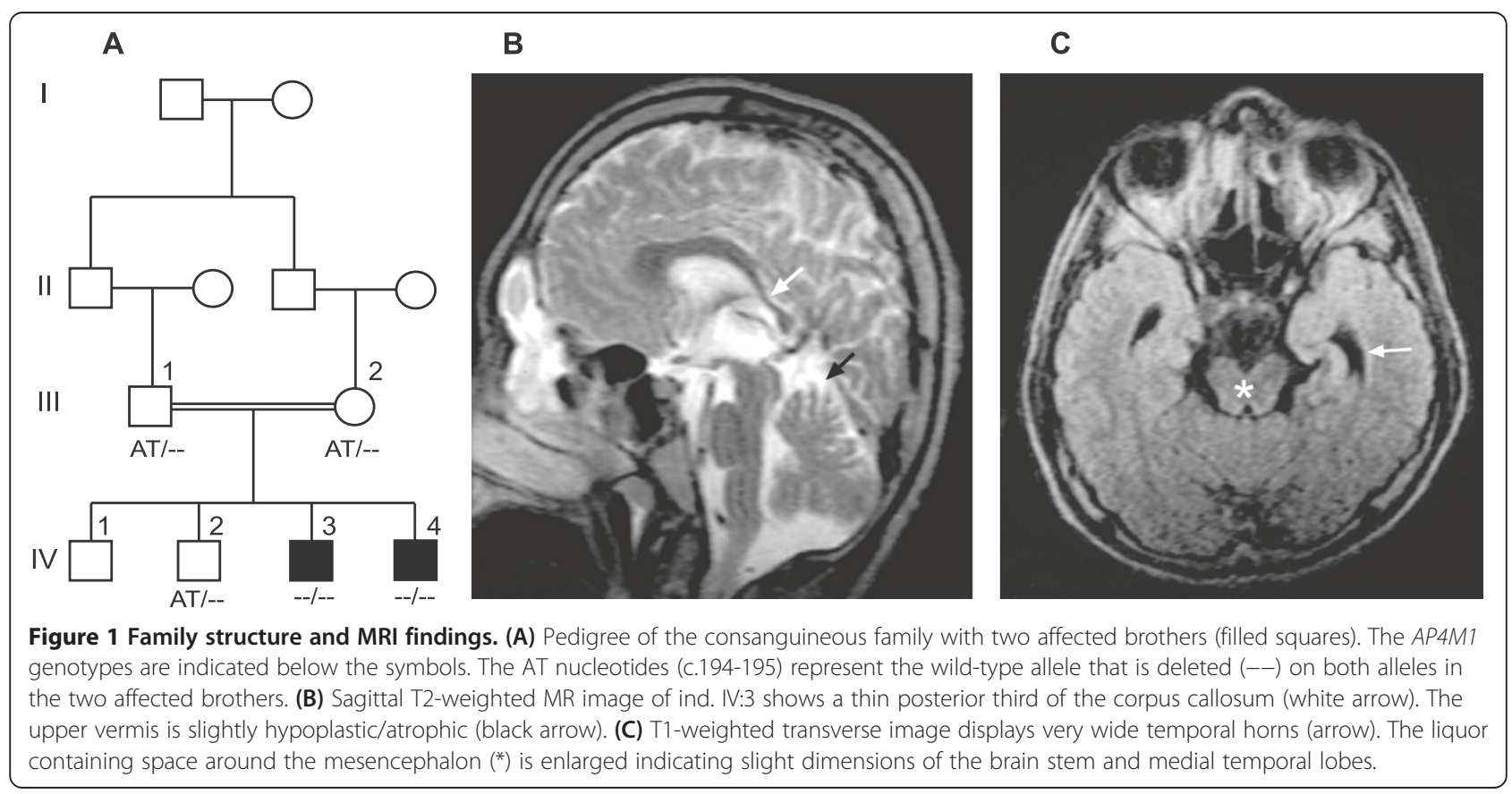


from ANNOVAR [17] and dbSNP135. Custom R scripts were used to identify potentially damaging variants that were shared between the patients while not present in any of the other $\sim 800$ exomes in the in-house database.

\section{PCR and mRNA analysis}

Segregation analysis of the AP4M1 variant c.194_195delAT, p.Y65Ffs 50 was performed by PCR and Sanger sequencing using standard protocols. Total RNA was extracted from cultured fibroblasts of two patients and two control individuals using the PureLink RNA Mini kit (Invitrogen), treated with DNA-free (Ambion) and then converted into cDNA by using RevertAid H Minus First Strand cDNA Synthesis Kit (Fermentas). Quantitative real-time PCR was performed with the Platinum SYBR Green qPCR SuperMixUDG kit (Invitrogen) then run and analyzed on the MxPro Real-Time PCR System (Stratagene). The primers for AP4M1 mRNA quantification were designed to amplify exon-intron boundaries between exons 2 and 4, respectively, to ensure that the amplicons were cDNA specific. All reactions were performed three times and in triplicates and normalized to $\beta$-actin mRNA. Student's two-tailed t-test was used for statistical analysis. Sanger sequencing was performed on the cDNA amplicon containing exon 3 in order to confirm the mutation. Primers sequences used to amplify the AP4M1 cDNA and AP4M1 exon 3 on genomic DNA were designed using Primer 3 Plus software (primer3plus.com/). All primer sequences are available upon request.

\section{Results}

\section{Clinical investigation}

Pregnancies and deliveries of both children were normal. Head circumference and muscle tonus were within normal ranges after delivery and during the first months of age. Hypotonia became evident in the elder brother (Ind. IV:3) at approximately 12 m.o.a and he learned to walk without support at 2 years of age. Spasticity developed gradually in the lower limbs with contractures of feet and positive Babinski sign. The ability to walk was lost at 6 years of age. Speech remained limited to a few single words and severe intellectual disability was evident at 3 years of age. He learned simple purposeful hand movements and he can feed himself. Seizures developed in childhood as well as an aggressive behavior in response to minor stimuli. Head circumference was -2SD at 14 years of age and facial dysmorphisms was otherwise restricted to a short philtrum (Table 1). MRI investigation, performed with a $0.23 \mathrm{~T}$ system at age 14 years of age showed a thin posterior corpus callosum, an enlarged third ventricle and widened temporal horns of the lateral ventricles. The mesencephalon and pons were thinner than expected for age. The upper vermis was hypoplastic/atrophic (Figure 1B-C). No abnormality in signal intensity was found.
Table 1 Clinical features of patients with AP4M1 mutations in this and previous studies

\begin{tabular}{|c|c|c|c|c|}
\hline \multirow[t]{2}{*}{ Parameters } & \multicolumn{2}{|c|}{ Present study } & \multirow{2}{*}{$\begin{array}{l}\text { Verkerk et al. } \\
\text { [16] }\end{array}$} & \multirow{2}{*}{$\begin{array}{l}\text { Tüysüz } \\
\text { et al. [15] }\end{array}$} \\
\hline & $\begin{array}{l}\text { Ind } \\
\text { IV:3 }\end{array}$ & $\begin{array}{l}\text { Ind } \\
\text { IV:4 }\end{array}$ & & \\
\hline Gender & $M$ & M & $3 \mathrm{~F} / 2 \mathrm{M}$ & $3 \mathrm{~F} / 1 \mathrm{M}$ \\
\hline $\begin{array}{l}\text { Age at last } \\
\text { examination (y.) }\end{array}$ & 14 & 12 & $1.5 / 21 / 22 / 23 / 24$ & $2.5 / 10.5 / 11 / 17$ \\
\hline Head circumference & $-2 S D$ & $-2 S D$ & -1 to $-2.5 \mathrm{SD}$ & -2 to -4 SD \\
\hline Height (cm) & 157 & 137 & NA & NA \\
\hline Intellectual disability & Severe & Severe & Severe in $5 / 5$ & Severe in $3 / 4$ \\
\hline Seizures & + & - & - & + \\
\hline Shy character & - & - & + & + \\
\hline Aggressive behavior & + & + & - & - \\
\hline Stereotype laughter & - & - & + & + \\
\hline $\begin{array}{l}\text { Severe speech } \\
\text { disorder }\end{array}$ & + & + & + & + \\
\hline Infantile hypotonia & + & + & + & + \\
\hline Hypertonia & + & + & + & + \\
\hline Hyperreflexia & + & + & NA & NA \\
\hline Babinski sign & + & + & + & NA \\
\hline Spasticity & + & + & + & + \\
\hline Club feet & - & - & + & + \\
\hline $\begin{array}{l}\text { Independent } \\
\text { walking (y.) }\end{array}$ & 2 & 4 & - & $+/-$ \\
\hline Ambulation & - & - & - & - \\
\hline \multicolumn{5}{|l|}{ Craniofacial features } \\
\hline Facial hypotonia & - & - & NA & + \\
\hline Bitemporal narrowing & - & - & NA & + \\
\hline Broad nasal bridge & - & - & NA & + \\
\hline Bulbous nose & - & + & NA & + \\
\hline Short philtrum & + & + & NA & + \\
\hline Brain MR imaging & Yes & No & $3 / 5$ & $4 / 4$ \\
\hline $\begin{array}{l}\text { Widened lateral } \\
\text { ventricles }\end{array}$ & + & & + & + \\
\hline $\begin{array}{l}\text { Thin splenium } \\
\text { of the CC }\end{array}$ & + & & + & + \\
\hline $\begin{array}{l}\text { Cerebellar } \\
\text { hypoplasia/atrophy }\end{array}$ & + & & $2 / 3$ & - \\
\hline
\end{tabular}

Abbreviations: + present, - absent, $S D$ standard deviation, $M$ male, $F$ female, NA: no data available, CC: corpus callosum, y:: years.

The second affected brother (ind. IV:4) was diagnosed with hypotonia at 12 months of age. Spasticity developed gradually in the lower limbs with contractures of feet and Babinski sign (Table 1). He learned to walk at 4 years of age but this ability was lost at 6 years of age. As for the elder brother, a severe cognitive deficit was evident with speech limited to a few single words, simple purposeful hand movement and aggressive behavior. Head circumference was -2SD at 12 years of age. Dysmorphic 
features included a short philtrum and a bulbous nose. The patient was not available for MRI investigation.

The upper limbs were unaffected in both brothers and none of them had pseudobulbar signs, impaired vision or hearing. Both brothers had a normal height, i.e. $157 \mathrm{~cm}$ and $137 \mathrm{~cm}$ at 14 years of age and 12 years of age, respectively.

\section{Whole exome sequencing}

Whole exome enrichment was performed on DNA samples from the two affected family members (ind. IV:3 and IV:4). After exome capture, the enriched DNA was sequenced using the Ion Proton system (Life technologies) and sequences were aligned to the human reference genome (hg19). An average of $97 \%$ of the exonic baits were covered at least $1 x$, and $90 \%$ were covered $>10 x$. Common variants were excluded by filtering against dbSNP130 (MAF >0.01) and 800 in house exome. Using filtered WES data from the two brothers, we identified altogether nine novel candidate variants. Five missense variants were located in the $\mathrm{X}$-chromosome genes $F A N C B, C C N B 3$, RGAG1, GPR50 and HAUS7, respectively. However, none of the amino acid substitutions were predicted as pathogenic and the five genes were not previously associated with the clinical features observed in our patients. Four autosomal and homozygous variants were identified in the TNFRSF14, AP4M1, RGMA and NINL genes. One of these variants is located in exon 3 of the AP4M1 gene (NM_004722.3) and consists of a two base pair deletion c.194_195delAT resulting in a frameshift p.Y65Ffs*50. With the previous knowledge on phenotypes associated with AP4M1 we performed Sanger sequencing and confirmed homozygosity for the AP4M1 deletion in the two affected family members. The unaffected parents and one healthy brother were heterozygous for the wild-type allele (Figure 2A). The eldest and healthy brother was not available for sampling. Furthermore, the two base pair deletion was excluded in 200 Swedish and 200 Pakistani control chromosomes and it was not present in AP4M1 sequences from 800 in house exomes, the dbSNP (www.ncbi.nlm. nih.gov/SNP/) or the EVS data release (ESP6500SI-V2) on the Exome Variant Server, NHLBI GO Exome Sequencing Project (ESP), Seattle, WA (URL: http://evs.gs. washington.edu/EVS/) when accessed March 2014. The variant c.194_195delAT is located in the region encoding a highly conserved longin-like domain (Figure 2B). Longin domains are globular structures found in different protein families that mediate protein-protein interactions in the membrane trafficking machinery [18].

\section{mRNA analysis}

To investigate the effect of the c.194_195delAT mutation at the transcript level we first sequenced the AP4M1 cDNA amplicon spanning exon 3 from both brothers and we detected only the mutated transcript. We then quantified the AP4M1 mRNA from cultured fibroblasts of the two affected family members (ind. IV:3 and ind. IV:4) as well as from control individuals using real-time qRT-PCR. The results indicated a 3 -fold reduction in AP4M1 mRNA expression in patient derived fibroblasts when compared to matched control fibroblasts $(p<0.0026$, Figure $2 \mathrm{C}$ ). The relative expression of AP4M1 in cases with respect to controls where calculated after normalization to $\beta$-actin.

\section{Discussion}

The phenotype in the two affected brothers of our family manifests as diplegic CP and severe intellectual disability. Autosomal recessive inheritance was likely because of the consanguineous and healthy parents. X-linked inheritance was possible but became highly unlikely when combining the results from bioinformatic analysis of $\mathrm{X}$-chromosome variants with the clinical observations. Instead, a search for homozygous variants in our WES data revealed four autosomal genes including the AP4M1 gene that is known to be associated with features observed in our patients. The AP4M1 mutation c.194_195delAT mutation results in a frame-shift and a premature stop p. Y65Ffs*50. Further analysis of the AP4M1 transcript in our patients showed that the deletion is associated with a three-fold reduction in AP4M1 mRNA levels presumably because of incomplete non-sense mediated mRNA decay $[19,20]$. The frame-shift mutation is positioned within a region encoding the highly conserved longin-like domain located in the more $\mathrm{N}$-terminal part of the protein. Longin domains are found in several protein families and serves as important regulators of membrane trafficking via protein-protein and intramolecular binding specificities [18]. Consequently, any translation of AP4M1 c.194_195delAT mRNA predicts a protein with a loss of membrane trafficking properties as well as a complete loss of a functional C-terminal cargo-binding domain [21]. Thus, the truncated AP4M1 protein would most likely be non-functional even if integrated in the AP-4 complex and with a resulting loss of complex integrity. In combination, the previous reports on AP-4 complex deficiency together with our genetic and clinical findings made the AP4M1 mutation a likely cause of the disease.

Our patients share several clinical features with other AP-4 deficient cases such as intellectual disability, reduced head circumference, spasticity and facial dysmorphisms, including a short philtrum and a bulbous nose [14]. Furthermore, the one individual available for MRI showed enlarged lateral ventricles and a thin corpus callosum that are typical findings reported previously in a few cases with mutations in each of the AP4B1, AP4M1 and AP4E1 genes, respectively $[8,11-15]$. In these previously reported patients signs of brain hypoplasia or atrophy are frequent but variable. Noteworthy, some clinical features in our 


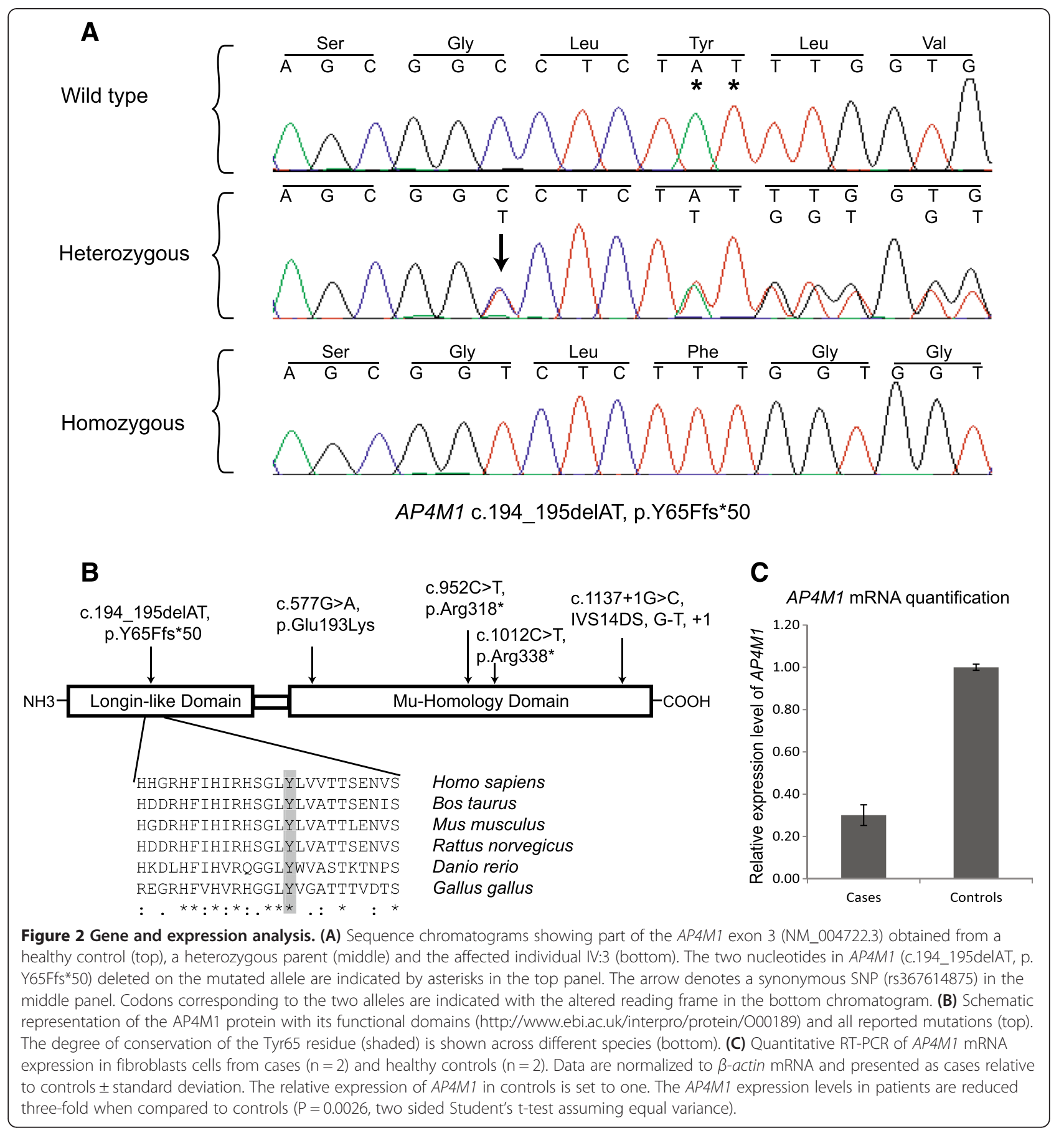

patients were different when compared to the few previously reported cases with mutations in the AP-4 subunit genes $[8,11-15]$. The clinical onset was relatively late, the body heights were within normal ranges for age and the upper limb functions were retained. Furthermore, the brothers have an aggressive behavior in response to minor stimuli in contrast to other patients with AP-4 deficiency. The reason for the clinical variation among $\mathrm{CP}$ patients carrying different mutations in AP4B1, AP4M1, AP4E1 and AP4S1 is unclear. It has been shown that the loss or structural change of a single AP-4 subunit disrupts the integrity of the entire AP-4 complex. Consequently, mutations in any of the AP-4 subunits would presumably have the same effects on vesicular glutamate receptor transport and neurotransmission resulting in similar clinical presentations. Therefore, one possible explanation for the clinical variability between individuals with mutations in genes encoding different AP-4 subunits is the effect 
from yet unknown modifier genes. This is further supported when comparing our observations with the two previously described families with truncating mutations in the AP4M1 subunit gene $[14,15]$.

Still, the AP-4 complex deficiency highlights two important aspects: It unravels a subgroup of patients with $\mathrm{CP}$ inherited as a Mendelian trait that can be recognized by a combination of appropriate diagnostic tools including WES. Second, AP-4 deficiency illustrates how a perturbed mechanism caused by interacting factors results in a clinical entity with $\mathrm{CP}$ and intellectual disability. In line with this, other factors and proteins involved in the AP-4 mediated vesicular trafficking may become strong candidates in the etiology of $\mathrm{CP}$.

\section{Conclusions}

We used whole-exome sequencing and identified a novel and truncating $A P 4 M 1$ mutation in two brothers with cerebral palsy and intellectual disability. Reduced levels of AP4M1 mRNA in the patients suggest incomplete nonsense mediated decay. The clinical investigation revealed aggressive behavior, normal body height, retained upper limb function and relatively late onset that add to the clinical variability in AP-4 deficiency. Still, the combination of hypotonia in infancy, minor facial dysmorphisms, reduced head circumference or microcephaly, para- or tetraplegia, severe intellectual disability and typical MRI findings should make AP-4 deficiency a conceivable diagnosis. Our study provides additional support for autosomal recessive inheritance in a subgroup of patients with $\mathrm{CP}$ and intellectual disability. Further studies are now needed to establish the frequency of AP-4 mutations and their variable clinical outcome.

\section{Abbreviations \\ AP-4: Adaptor protein 4 complex; AP4M1: Adaptor protein 4 complex subunit M1; CP: Cerebral palsy; WES: Whole exome sequencing; MRI: Magnetic resonance imaging; RT-PCR: Reverse transcription polymerase chain reaction; AMPA: a-amino-3-hydroxy-5-methyl-4-isoxazolepropionic acid; CDNA: Complementary DNA; mRNA: Messenger RNA; SNP: Single nucleotide polymorphism; SD: Standard deviation; MAF: Mean allele frequency.}

\section{Competing interests}

The authors declare that they have no competing interests.

\section{Authors' contributions}

$\mathrm{JK}, \mathrm{SMB}$ and ND designed and supervised the study. NAM and MT collected blood and skin samples. AM, SSW, UA and TNK performed clinical investigations and DNA extractions. MJ and JK carried out analysis of filtered whole exome sequence data and performed Sanger sequencing, fibroblast cell culture, RNA extraction and qRT-PCR. RR analyzed the MR images. ND wrote the manuscript together with MJ. All authors read and approved the final manuscript.

\section{Acknowledgments}

We acknowledge the family members for their cooperation in the study and we are indepted to Dr. Atle Melberg for discussions. This work was supported by grants from the Swedish Research Council (K2013-66X-10829-20-3), Uppsala University Hospital, Uppsala University and the Science for Life Laboratory. M.J. was supported by the Higher Education Commission of Pakistan and J.K was supported by the Swedish Society for Medical Research.

\section{Author details}

${ }^{1}$ Human Molecular Genetics Laboratory, National Institute for Biotechnology and Genetic Engineering (NIBGE), PIEAS, Faisalabad 38000, Pakistan. 2Department of Immunology, Genetics and Pathology, Science for Life Laboratory, Uppsala University, Uppsala 751 08, Sweden. ${ }^{3}$ Department of Radiology, Uppsala University, Uppsala 751 85, Sweden.

Received: 30 June 2014 Accepted: 3 December 2014

Published online: 14 December 2014

\section{References}

1. Clark SL, Hankins GD: Temporal and demographic trends in cerebral palsy-fact and fiction. Am J Obstet Gynecol 2003, 188(3):628-633.

2. Pakula AT, Van Naarden BK, Yeargin-Allsopp M: Cerebral palsy: classification and epidemiology. Phys Med Rehabil Clin N Am 2009, 20(3):425-452.

3. Costeff H: Estimated frequency of genetic and nongenetic causes of congenital idiopathic cerebral palsy in west Sweden. Ann Hum Genet 2004, 68(Pt 5):515-520.

4. Graham EM, Ruis KA, Hartman AL, Northington FJ, Fox HE: A systematic review of the role of intrapartum hypoxia-ischemia in the causation of neonatal encephalopathy. Am J Obstet Gynecol 2008, 199(6):587-595.

5. Petterson B, Stanley F, Henderson D: Cerebral palsy in multiple births in Western Australia: genetic aspects. Am J Med Genet 1990, 37(3):346-351.

6. Blair E, Al Asedy F, Badawi N, Bower C: Is cerebral palsy associated with birth defects other than cerebral defects? Dev Med Child Neurol 2007, 49(4):252-258.

7. Garne E, Dolk H, Krageloh-Mann I, Holst Ravn S, Cans C: Cerebral palsy and congenital malformations. Eur J Paediatr Neurol 2008, 12(2):82-88.

8. Moreno-De-Luca A, Helmers SL, Mao H, Burns TG, Melton AM, Schmidt KR, Fernhoff PM, Ledbetter DH, Martin CL: Adaptor protein complex-4 (AP-4) deficiency causes a novel autosomal recessive cerebral palsy syndrome with microcephaly and intellectual disability. J Med Genet 2011, 48(2):141-144

9. Hirst J, Irving C, Borner GH: Adaptor protein complexes AP-4 and AP-5: new players in endosomal trafficking and progressive spastic paraplegia. Traffic 2013, 14(2):153-164.

10. Matsuda S, Yuzaki M: Polarized sorting of AMPA receptors to the somatodendritic domain is regulated by adaptor protein AP-4. Neurosci Res 2009, 65(1):1-5.

11. Abdollahpour H, Alawi M, Kortum F, Beckstette M, Seemanova E, Komarek V, Rosenberger $G$, Kutsche K: An AP4B1 frameshift mutation in siblings with intellectual disability and spastic tetraplegia further delineates the AP-4 deficiency syndrome. Eur J Hum Genet 2014, Advance online publication.

12. Abou Jamra R, Philippe O, Raas-Rothschild A, Eck SH, Graf E, Buchert R, Borck G, Ekici A, Brockschmidt FF, Nothen MM, Munnich A, Strom TM, Reis A, Colleaux $L$ : Adaptor protein complex 4 deficiency causes severe autosomal-recessive intellectual disability, progressive spastic paraplegia, shy character, and short stature. Am J Hum Genet 2011, 88(6):788-795.

13. Najmabadi H, Hu H, Garshasbi M, Zemojtel T, Abedini SS, Chen W, Hosseini M, Behjati F, Haas S, Jamali P, Zecha A, Mohseni M, Püttmann L, Vahid LN, Jensen C, Moheb LA, Bienek M, Larti F, Mueller I, Weissmann R, Darvish H, Wrogemann K, Hadavi V, Lipkowitz B, Esmaeeli-Nieh S, Wieczorek D, Kariminejad R, Firouzabadi SG, Cohen M, Fattahi Z: Deep sequencing reveals 50 novel genes for recessive cognitive disorders. Nature 2011, 478(7367):57-63.

14. Tuysuz B, Bilguvar K, Kocer N, Yalcinkaya C, Caglayan O, Gul E, Sahin S, Comu S, Gunel M: Autosomal recessive spastic tetraplegia caused by AP4M1 and AP4B1 gene mutation: Expansion of the facial and neuroimaging features. Am J Med Genet A 2014, 164:1677-1685.

15. Verkerk AJ, Schot R, Dumee B, Schellekens K, Swagemakers S, Bertoli-Avella AM, Lequin MH, Dudink J, Govaert P, van Zwol AL, Hirst J, Wessels MW, Catsman-Berrevoets C, Verheijen FW, de Graaff E, de Coo IF, Kros JM, Willemsen R, Willems PJ, van der Spek PJ, Mancini GM: Mutation in the AP4M1 gene provides a model for neuroaxonal injury in cerebral palsy. Am J Hum Genet 2009, 85(1):40-52.

16. Khan TN, Klar J, Tariq M, Anjum Baig S, Malik NA, Yousaf R, Baig SM, Dahl N: Evidence for autosomal recessive inheritance in SPG3A caused by homozygosity for a novel ATL1 missense mutation. Eur J Hum Genet 2014, 22:1180-1184.

17. Wang K, Li M, Hakonarson H: ANNOVAR: functional annotation of genetic variants from high-throughput sequencing data. Nucleic Acids Res 2010, 38(16):e164 
18. Rossi V, Banfield DK, Vacca M, Dietrich LE, Ungermann C, D'Esposito M, Galli T, Filippini F: Longins and their longin domains: regulated SNAREs and multifunctional SNARE regulators. Trends Biochem Sci 2004, 29 (12):682-688.

19. Xiao J, Nance MA, LeDoux MS: Incomplete nonsense-mediated decay facilitates detection of a multi-exonic deletion mutation in SGCE. Clin Genet 2013, 84(3):276-280.

20. Magyar I, Colman D, Arnold E, Baumgartner D, Bottani A, Fokstuen S, Addor MC Berger W, Carrel T, Steinmann B, Mátyás G: Quantitative sequence analysis of FBN1 premature termination codons provides evidence for incomplete NMD in leukocytes. Hum Mutat 2009, 30(9):1355-1364.

21. Owen DJ, Evans PR: A structural explanation for the recognition of tyrosine-based endocytotic signals. Science 1998, 282(5392):1327-1332.

doi:10.1186/s12881-014-0133-2

Cite this article as: Jameel et al: A novel AP4M1 mutation in autosomal recessive cerebral palsy syndrome and clinical expansion of AP-4 deficiency. BMC Medical Genetics 2014 15:133.

\section{Submit your next manuscript to BioMed Central and take full advantage of:}

- Convenient online submission

- Thorough peer review

- No space constraints or color figure charges

- Immediate publication on acceptance

- Inclusion in PubMed, CAS, Scopus and Google Scholar

- Research which is freely available for redistribution 